\title{
siRNA-mediated silencing of phosphodiesterase 4B expression affects the production of cytokines in endotoxin-stimulated primary cultured microglia
}

\author{
HAO CHENG, ZHIFANG WU, XIAOYUN HE, QINGZHEN LIU, HONGBIN JIA, YAN DI and QING JI \\ Department of Anesthesiology, Jinling Hospital, School of Medicine, Nanjing University, \\ Nanjing, Jiangsu 210002, P.R. China
}

Received April 28, 2015; Accepted May 19, 2016

DOI: $10.3892 / \mathrm{etm} .2016 .3575$

\begin{abstract}
Phosphodiesterase 4 (PDE4) has four subtypes: PDE4A, PDE4B, PDE4C and PDE4D. The expression of PDE4 subtypes in microglial cells and the specific contribution of each subtype to inflammation remain unclear. In this study, the expression of PDE4 subtypes in primary microglial cells was assayed. Primary microglial cells were then transfected with specific small interfering RNA (siRNA) against each PDE4 subtype. PDE4 subtype A-D knockdown was confirmed by quantitative polymerase chain reaction. Secreted cytokines in the supernatant and intracellular cyclic adenosine monophosphate (cAMP) levels of transfected cells were measured. The effect of PDE4B siRNA on the activation of extracellular regulated protein kinase (ERK) induced by lipopolysaccharide (LPS) in microglia was further tested by western blotting. Results showed that the primary microglial cells expressed all four types of PDE4s at the protein level. Transfection with the four siRNAs inhibited PDE4 subtype A-D mRNA expression, respectively. In primary microglial cells, treatment with PDE4B siRNA significantly inhibited the expression of tumor necrosis factor- $\alpha$ and interleukin (IL)-1 $\beta$, and enhanced the expression of cAMP, while siRNAs to other subtypes had no significant effects. However, none of the four siRNAs had any significant effect on the expression of IL-10. Furthermore, in the PDE4B group, the level of phosphorylated ERK was reduced. Among the four PDE4 subtypes, PDE4B plays an important role in regulating inflammatory responses in microglia, potentially through initially regulating the intracellular cAMP concentration.
\end{abstract}

Correspondence to: Professor Qing Ji, Department of Anesthesiology, Jinling Hospital, School of Medicine, Nanjing University, 305 East Zhongshan Road, Nanjing, Jiangsu 210002, P.R. China

E-mail: jzwrite@163.com

Key words: phosphodiesterase 4B, small interfering RNA, microglia, inflammation

\section{Introduction}

Microglial cells, which comprise $12 \%$ of the cell population in the central nervous system (CNS), are macrophage-like cells (1). Although they have been recognized mainly for their scavenging functions in the brain, certain studies have suggested that inflammation mediated by microglia contributes to the development of neurodegenerative diseases, such as Alzheimer's disease, multiple sclerosis and neuropathic pain (2-4). Microglial cells, once activated during brain pathologies, release a variety of neurotoxic substances including nitric oxide, cytotoxic cytokines, tumor necrosis factor- $\alpha$ (TNF- $\alpha$ ) and interleukin (IL)-1 $\beta$. As a result of their remarkable ability to respond rapidly to a changing external environment and participate in the regulation of the innate and adaptive immune system in the CNS, microglia have become a prime target for therapeutic intervention in a variety of CNS insults $(5,6)$.

Previous studies have demonstrated that a rise in the intracellular 3',5'-cyclic adenosine monophosphate (cAMP) level leads to the suppression of the activities and proliferation of microglial cells $(7,8)$. Among the various cAMP-elevating agents, inhibitors of phosphodiesterase type 4 (PDE4) deserve increased attention because they increase the cAMP level in mononuclear cells or macrophages more effectively than other types of PDE inhibitors (9). Recent studies in mammals have revealed that PDE4 is a family of cAMP-specific PDEs, with $>20$ isozymes that are encoded by 4 genes (PDE4A-D) $(10,11)$. The four subfamilies share a conserved catalytic domain and upstream conserved region, which are upstream conserved region (UCR)1 and UCR2 motifs of the regulatory domain. Therefore, the developed inhibitors often are not able to target a subfamily specifically. Clinical evaluations in various inflammatory conditions have revealed that the anti-inflammatory effect of PDE4 inhibitors has a very broad spectrum, affecting almost all inflammatory cells (12-14). Some adverse effects that are associated with this lack of specificity, such as nausea and emesis, limit the dosage level of those inhibitors and prevent them from achieving an ideal level of immunomodulatory activity (15).

RNA interference (RNAi) is a method that is able to directly eliminate the function of a targeted gene. Studies 
performed on mammals have demonstrated the superiority of silencing induced by small interfering RNAs (siRNAs) to the traditional antisense molecules $(16,17)$. Therefore, in recent years, RNAi has been established as a preferred technology for investigating and validating protein function. More importantly, the high specificity of RNAi provides the potential to selectively silence a subtype from a family of closely related genes (18).

PDE4 inhibitors have already been shown to increase the cAMP level in microglial cells (19), but it is not clear which particular subtype is responsible for such increase. The present study was designed to first study whether microglial cells actually express the four PDE4 subtypes. The RNAi technique was then applied to silence different subtypes of the PDE4 gene, and the specific effects of each subtype on the production of cytokines in endotoxin-stimulated primary cultured microglia were observed.

\section{Materials and methods}

Materials. Lipopolysaccharide (LPS) from Escherichia coli 011:B4 (Santa Cruz Biotechnology, Inc., Dallas, TX, USA) was suspended in phosphate-buffered saline (PBS) at a concentration of $20 \mathrm{mg} / \mathrm{ml}$ and stored at $-20^{\circ} \mathrm{C}$. Primers for reverse transcription-quantitative polymerase chain reaction (RT-qPCR) were synthesized by Invitrogen (Thermo Fisher Scientific, Inc., Waltham, MA, USA). Antibodies for western blot analysis were: i) Anti-PDE4A (ab14607; 1:1,000), anti-PDE4B (ab14611; 1:1,000), anti-PDE4D (ab14613; 1:500) and anti-CD11b (ab75476; 1:1,000) antibodies (all purchased from Abcam, Cambridge, MA, USA); ii) anti-PDE4C antibody (1:300; PD4C-301AP; Fabgennix International, Inc., Frisco, TX, USA); iii) anti-p44/42 mitogen-activated protein kinase [4695; 1:1,000; MAPK; extracellular signal-regulated kinase (ERK)1/2; Cell Signalling Technology, Inc., Danvers, MA, USA], anti-phosphorylated (P)-p44/42 MAPK (T202/Y204; 14474; 1:1,000; Cell Signalling Technology, Inc.), and anti-rabbit IgG horseradish peroxidase (HRP)-linked antibody (ab6721; 1:1,000; Cell Signaling Technology, Inc.). Except where indicated, all other materials were from Invitrogen (Thermo Fisher Scientific, Inc.).

Micoglial culture. Microglia were isolated from primary cultures of rat brains by the method previously described with slight modifications (20). Briefly, two whole brains of Sprague-Dawley rats (Experimental Animal Center of Jinling Hospital, Nanjing, China) at postnatal days 1-3 were used for each flask (Delta-treated flask, $75 \mathrm{~cm}^{2}$; Nunc ${ }^{\mathrm{TM}}$ ). Following the dissection of the brains and careful stripping of the meninges, brains were triturated with a Pasture pipette with $3 \mathrm{ml}$ Dulbecco's modified Eagle's medium (DMEM) containing $10 \%$ fetal calf serum, $2 \mathrm{mM}$ L-glutamine, $100 \mathrm{U} / \mathrm{ml}$ penicillin and $100 \mu \mathrm{g} / \mathrm{ml}$ streptomycin. The solution was centrifuged at $800 \mathrm{xg}$ for $10 \mathrm{~min}$. The precipitates were suspended completely with $2 \mathrm{ml}$ DMEM and were plated in the aforementioned flask. These mother cultures were maintained in a $5 \% \mathrm{CO}_{2}$ humidified incubator at $37^{\circ} \mathrm{C}$ and the medium was exchanged every 3 days after the initial seeding. After 9-10 days, many round and phase-bright microglia were isolated by shaking for $4 \mathrm{~h}$ at $40 \mathrm{xg}$. Floating cells in the supernatant were collected, centrifuged and disseminated onto 24-well plates (Corning Incorporated, Corning, NY, USA; $5 \times 10^{5}$ cells/well) or 96-well plates (Corning Incorporated; 5x10 $10^{4}$ cells/well). After $20 \mathrm{~min}$, the medium was changed and the cells were washed twice with PBS to remove non-adherent cells. The remaining microglia were allowed to stabilize for 1 day in DMEM containing $10 \%$ fetal bovine serum (FBS) prior to use. In this culture, the purity of the microglia was $95-98 \%$ as determined using an anti-CD11b antibody (21).

Western blot analysis. Western blotting was performed for the analysis of the expression of PDE4 subtypes A-D at the protein level. Briefly, protein samples (cell extracts, $50 \mathrm{mg}$ protein) were separated by a $10 \%$ sodium dodecyl sulfate-polyacrylamide gel and transferred to $0.45-\mu \mathrm{m}$ polyvinylidene difluoride membranes. The membranes were blocked with $5 \%$ fat-free dry milk dissolved in Tris-buffered saline $(\mathrm{NaCl}, 0.138 \mathrm{M} ; \mathrm{KCl}, 0.0027 \mathrm{M}, \mathrm{pH}$ 8.0) plus $0.1 \%$ Tween-20 (TBST) and incubated overnight with specific antibodies against PDE4 subtypes A-D (1:1,000). After extensive washing with TBST, the membranes were incubated with anti-rabbit IgG HRP-linked antibody solution $(1: 1,000)$ for $1 \mathrm{~h}$ at room temperature. The proteins were visualized using chemiluminescence reagents provided with an ECL kit (Amersham; GE Healthcare Bio-Sciences, Pittsburgh, PA, USA) and exposure to film. Scanning densitometry using Image J 2X (Rawak Software Inc., Stuttgart, Germany) was used for semi-quantitative analysis of the data.

siRNA preparation. The siRNAs were designed based on the rat PDE4A (Rattus norvegicus, PDE4A, NM_013101), PDE4B (Rattus norvegicus, PDE4B, NM_017031), PDE4C (Rattus norvegicus, PDE4C, XM_001070301) and PDE4D (Rattus norvegicus, PDE4D, NM_017032) using BLOCK-iT RNAi Designer (Invitrogen; Thermo Fisher Scientific, Inc.). An siRNA target sequence was selected for each of the subtypes. One non-specific siRNA (mismatch RNA) was also selected. Sequences of the siRNAs used in the present study are summarized in Table I.

Transfection of siRNAs into microglia. Primary microglial cells were randomly divided into eight groups: Control group (no LPS, no transfection), LPS group (no transfection), and transfection groups, including vehicle group (transfected with Lipofectamine ${ }^{\mathrm{TM}}$ RNAiMAX reagent; Thermo Fisher Scientific, Inc.), mismatch group (transfected with mismatch siRNA) and PDE4-siRNA groups A-D (transfected with siRNA targeting PDE4 subtypes A-D, respectively).

To transfect the vehicle (Lipofectamine RNAiMAX reagent), mismatch RNA, PDE4A, PDE4B, PDE4C or PDE4D stealth siRNAs into microglia, respectively, 1 day prior to transfection, microglia were cultured in DMEM without antibiotics and FBS. For each transfection in 24-well plates, $20 \mathrm{nM}$ mismatch RNA, PDE4A, PDE4B, PDE4C or PDE4D stealth siRNA was diluted in $100 \mu 1$ DMEM without antibiotics and FBS and gently mixed with $1 \mu$ l Lipofectamine RNAiMAX reagent according to the manufacturer's protocol. Following incubation for $15 \mathrm{~min}$ at room temperature, transfection mixture was added to each well and applied to each 
Table I. Sequences of the siRNAs used in the present study.

\begin{tabular}{lll}
\hline siRNA & Strand & Sequence (5' to 3') \\
\hline PDE4A & Sense & AAGAGUGAGAAGUUGCUUCGAACGC \\
& Antisense & GCGUUCGAAGCAACUUCUCACUCUU \\
PDE4B & Sense & UCUUCUUGCAGGCUGACUCUGGUGA \\
& Antisense & UCACCAGAGUCAGCCUGCAAGAAGA \\
PDE4C & Sense & AGGAAAGUCUGCGAGAUGUACUCUG \\
& Antisense & CAGAGUACAUCUCGCAGACUUUCCU \\
PDE4D & Sense & AUGGAUGGUUGGUUGCACAUGGGUG \\
& Antisense & CACCCAUGUGCAACCAACCAUCCAU \\
\hline
\end{tabular}

siRNA, small interfering RNA; PDE, phosphodiesterase.

Table II. PCR primers used in the present study.

\begin{tabular}{lll}
\hline Primer & Direction & Sequence $\left(5^{\prime}\right.$ to $\left.3^{\prime}\right)$ \\
\hline PDE4A & Forward & GAAGACAACCGGGACTCCT \\
& Reverse & CCTCAGTGGTAGGCAATCC \\
PDE4B & Forward & CCTCCGACACCTTCGTAAC \\
& Reverse & CCAGGTCTGTGAAGACAGC \\
PDE4C & Forward & GAAGGGCACTACCACTCCA \\
& Reverse & GTGTATAGCGCACGCAAAGA \\
PDE4D & Forward & CCCTCTTGACTGTTATCATGCACACC \\
& Reverse & GATCCTACATCATGTATTGCACTGGC \\
GAPDH & Forward & CCATGTTCGTCATGGGTGTGAACCA \\
& Reverse & GCCAGTAGAGGCAGGGATGATGTTC
\end{tabular}

PCR, polymerase chain reaction; PDE, phosphodiesterase; GAPDH, glyceraldehyde 3-phosphate dehydrogenase.

well in a total volume of $500 \mu 1$ DMEM. Transfected cells were further incubated at $37^{\circ} \mathrm{C}$ for $48 \mathrm{~h}$ before assays were carried out.

$R T$-qPCR analysis. RT-qPCR was performed to detect the expression of PDE4A, B, C and D genes. Total RNA was isolated from cells using TRIzol reagent and a spectrophotometer was used to determine the quality and quantity of the RNA. TRIzol reagent was used according to the manufacturer's instructions. In brief, a total of $250 \mu \mathrm{l}$ TRIzol reagent was added into the well $\left(5 \times 10^{5}\right.$ cells/well) to lyse the cells by pipetting them up and down several times after removing the growth media from the well. Next, $50 \mu \mathrm{l}$ chloroform was added after incubating the homogenized sample for $5 \mathrm{~min}$ in the tube at room temperature. Following vigorous shaking for $15 \mathrm{sec}$ and incubation at room temperature for $3 \mathrm{~min}$, the sample was centrifuged at $12,000 \mathrm{x}$ g for $15 \mathrm{~min}$ at $4^{\circ} \mathrm{C}$. The aqueous phase of the sample was removed into a new tube, then incubated with $125 \mu \mathrm{l}$ isopropanol at room temperature for $10 \mathrm{~min}$. The total RNA was precipitated by centrifugation at $12,000 \mathrm{xg}$ for $15 \mathrm{~min}$ at $4^{\circ} \mathrm{C}$. The RNA pellet was washed with $250 \mu 175 \%$ ethanol, then centrifuged at 7,500xg for $5 \mathrm{~min}$ at $4^{\circ} \mathrm{C}$. After discarding the wash, the RNA pellet was air dried for 8 min. Finally, the RNA pellet was suspended in RNase-free water $(20 \mu \mathrm{l})$. The isolated RNA was stored at $-70^{\circ} \mathrm{C}$ before RT-qPCR. The quality and quantity of RNA were determined using a spectrophotometer by measuring the OD levels at $260 \mathrm{~nm}$ and $280 \mathrm{~nm}$, respectively. The ratios of OD 260/OD 280 for the samples were between 1.8-2.0, which indicated the purity of the samples was quite good. Thus we did not treat the isolated RNA before RT-qPCR.

cDNA was synthesized from the RNA using reverse transcription. The reverse transcription (RT) reaction was performed in a $20 \mu \mathrm{l}$ total reaction volume containing $4 \mu \mathrm{l}$ of 5X RT buffer, $4 \mu \mathrm{l}$ of $2.5 \mathrm{mM}$ dNTPs, $1 \mu \mathrm{l}$ of Multiscribe reverse transcriptase $(50 \mathrm{U} / \mu \mathrm{l})$ (Promega, Madison, WI, USA), $1 \mu \mathrm{l}$ of RNase inhibitor, $5 \mu \mathrm{l}$ of RNase-free water and $3 \mu \mathrm{g}$ of isolated total RNA in a $5 \mu \mathrm{l}$ volume. The RT reaction was performed at $25^{\circ} \mathrm{C}$ for $10 \mathrm{~min}, 37^{\circ} \mathrm{C}$ for $120 \mathrm{~min}$ and $95^{\circ} \mathrm{C}$ for $5 \mathrm{~min}$. qPCR analysis was performed using a 7500 Fast Real-Time PCR System (Applied Biosystems; Thermo Fisher Scientific, Inc.). qPCR was performed in a final volume of $20 \mu \mathrm{l}$, containing $10 \mu \mathrm{M}$ each primer, $1 \mu \mathrm{l}$ SYBR green (20X) and $1 \mu$ l template. The program profile was as follows: $95^{\circ} \mathrm{C}$ for $2 \mathrm{~min}, 40$ cycles of denaturation for $10 \mathrm{sec}$ at $95^{\circ} \mathrm{C}$, annealing for $30 \mathrm{sec}$ at $60^{\circ} \mathrm{C}$ and extension 
for $30 \mathrm{sec}$ at $70^{\circ} \mathrm{C}$. The PCR primers used in this study are summarized in Table II.

The $\Delta \Delta \mathrm{Cq}$ method was used to normalize the qPCR data, and the level of GADPH mRNA was utilized as a reference. The relative expression level of target $\mathrm{mRNA}$ was calculated according to the difference between the reference and target $\mathrm{Cq}$ values for each sample. Next, the level of target mRNA in groups with different treatment was normalized by setting the level in the control group as 1 .

Analysis of cytokines and $c A M P$. For measuring the production of TNF- $\alpha$, IL-1 $\beta$, IL-10 and cAMP, primary microglial cells were randomly divided into the eight aforementioned groups. Following transfection and $48 \mathrm{~h}$ of incubation, the culture medium was discarded and LPS was added at final concentration of $100 \mathrm{ng} / \mathrm{ml}$. The supernatants of the cultured microglia were collected after 2, 4, 6, 12 and $24 \mathrm{~h}$ of stimulation by LPS.

The intracellular cAMP level in the microglia was detected following $30 \mathrm{~min}$ of stimulation by LPS. Briefly, cells were washed three times in cold PBS and resuspended in cell lysis buffer. Cells were frozen and thawed with gentle mixing twice. Cell lysates were then centrifuged at $600 \mathrm{xg}$ for $10 \mathrm{~min}$ to remove cellular debris. The above supernatants and cell lysates were stored at $-80^{\circ} \mathrm{C}$ before assaying.

ELISA kits for TNF- $\alpha$, IL- $1 \beta$, IL-10 and cAMP determination were purchased from R\&D Systems, Inc. (Minneapolis, MN, USA) and used to determine the levels of these proteins according to the manufacturer's protocol. Each experiment was performed in triplicate.

Detection of ERK protein expression using western blot analysis. At $24 \mathrm{~h}$ after stimulation by LPS, cells from the control, LPS, vehicle, mismatch RNA and PDE4B groups were collected and the expression levels of total ERK and p-ERK protein were assessed by western blotting as described above. The primary antibodies were p44/42 MAPK (ERK1/2) $(1: 1,000)$ and P-p44/42 MAPK (T202/Y204) (1:1,000).

Statistical analysis. Results are expressed as the mean \pm standard deviation of at least three independent determinations. SPSS statistical software version 13.0 was used for data analysis. Data from the RT-qPCR and western blot experiments were accomplished using one-way analysis of variance (ANOVA). Data from the ELISA were analyzed using repeated-measures ANOVA. Least-significance difference post-hoc comparison was used to detect significant differences for the ANOVA analyses. $\mathrm{P}<0.05$ was considered to indicate a statistically significant result.

\section{Results}

Expression levels of PDE4 subtypes A-D in microglia. The expression of PDE4 subtypes A-D in microglia were studied at the protein level. The protein expression levels of PDE4 subtypes A-D were determined by western blot analysis (Fig. 1). The results demonstrated that the microglia expressed all four subtypes of PDE4.

Expression levels of PDE4 subtypes $A-D$ are reduced by siRNAs. To investigate the potential function of the four PDE4 subtypes in microglia, siNRAs were used to knock down endogenous PDE4A-D gene expression. A siRNA sequence was selected for each of the subtypes A-D. The expression of PDE4A at the mRNA level was reduced to $36.6 \pm 5.2 \%$ of that in the control group $(\mathrm{P}<0.05$; Fig. $2 \mathrm{~A})$. The expression of PDE4B, PDE4C and PDE4D at the $\mathrm{mRNA}$ level was reduced to $34.7 \pm 6.4,33.2 \pm 4.8$ and $27.5 \pm 7.0 \%$ of that in the control group, respectively ( $\mathrm{P}<0.05$; Fig. 2B-D). However, no significant differences were observed among the LPS, mismatch RNA and vehicle groups $(\mathrm{P}>0.05)$.

Effects of PDE4 subtype A-D siRNAs on the LPS-induced release of cytokines. To study the effects of silencing PDE4 subtypes A-D with siRNA on the LPS-induced release of cy tokines, the levels of TNF- $\alpha$, IL- $1 \beta$ and IL-10 were measured by ELISA. First, in the LPS group, microglia showed a strong increase in TNF- $\alpha$ production at $2 \mathrm{~h}$ after stimulation with LPS, then the amount increased markedly and the maximum was observed at $6 \mathrm{~h}$. After that, the amount of TNF- $\alpha$ in the medium decreased gradually. Among the groups treated with siRNAs, only PDE4B siRNA significantly inhibited the production of TNF- $\alpha$ compared with the level in the LPS group ( $\mathrm{P}<0.05$; Fig. 3A). Second, in the LPS group a considerable amount of IL-1 $\beta$ was detected at $4 \mathrm{~h}$. The maximum level was reached at $6 \mathrm{~h}$, and this level was maintained for $24 \mathrm{~h}$. Among the groups treated with siRNAs, only the PDE4B group showed a significantly reduced level of IL-1 $\beta$ $(\mathrm{P}<0.05$; Fig. 3B). Third, in contrast to TNF- $\alpha$ and IL-1 $\beta$, the levels of IL-10 were not affected by any of the siRNAs when compared with the LPS group ( $\mathrm{P}>0.05$; Fig. 3C). These data demonstrate that the action of PDE4B-siRNA in primary microglia is highly selective, inhibiting the production of TNF- $\alpha$ and IL-1 $\beta$ but not enhancing the production of IL-10.

Effects of siRNA of PDE4 subtypes A-D on the intracellular $c A M P$ level. To test which subtype of PDE4 is potentially responsible for the regulation of the intracellular cAMP level, lysates were collected to determination of cAMP level after 30 min of stimulation by LPS (Fig. 4). Prior to stimulation with LPS, no clear differences were observed in cAMP levels among the eight groups. LPS stimulation significantly increased the cAMP levels in all groups compared with those in the control group $(\mathrm{P}<0.05)$. However, only the PDE4B-siRNA group showed a significantly higher intracellular cAMP level than that in the LPS group $(\mathrm{P}<0.05)$. This indicates that $\mathrm{PDE} 4 \mathrm{~B}$ is pivotal in the regulation of the cAMP level in microglial cells.

Effect of PDE4B-siRNA on the LPS-induced activation of ERK in microglia. ERK is known to be involved in the regulation of cytokine expression by influencing the activities of several transcriptional factors $(22,23)$. The effects of PDE4B on ERK activities in microglial cells were examined by western blotting. Treatment with LPS caused significant increases in the p-ERK levels in the microglial cells compared with those in the control group, and PDE4B-siRNA significantly suppressed the elevation of the p-ERK level induced by LPS treatment $(\mathrm{P}<0.05$; Fig. 5A). No significant differences in the total ERK levels were observed among all groups ( $\mathrm{P}>0.05$; Fig. 5B). 
A

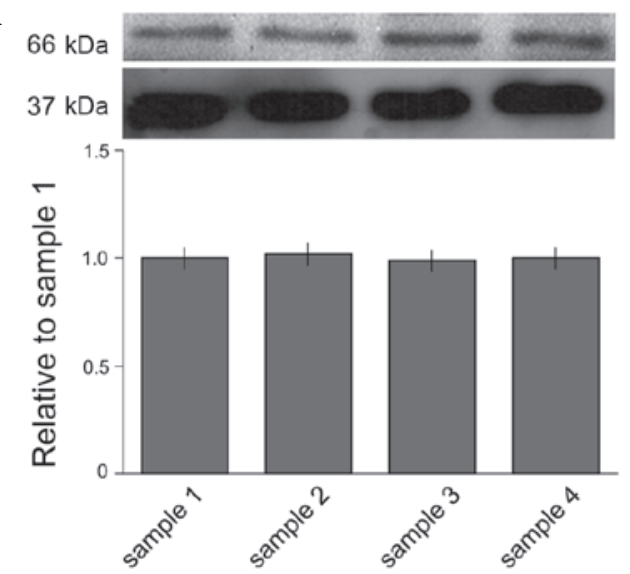

C
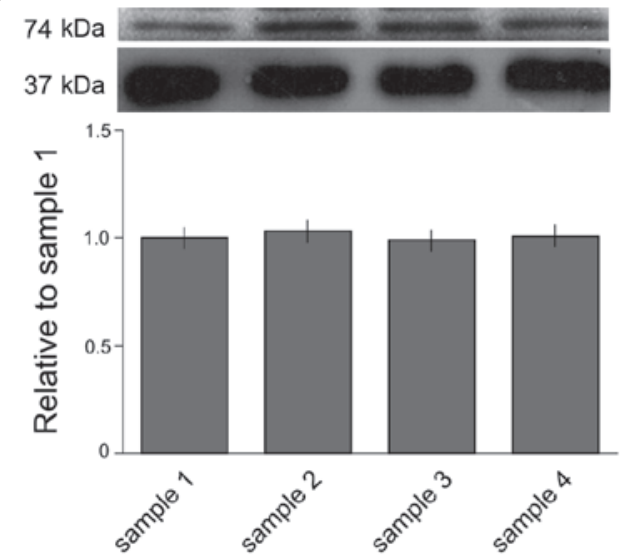

B

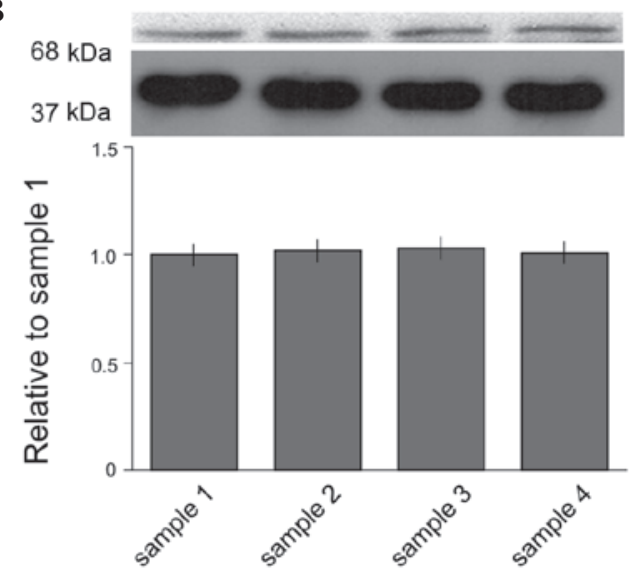

D
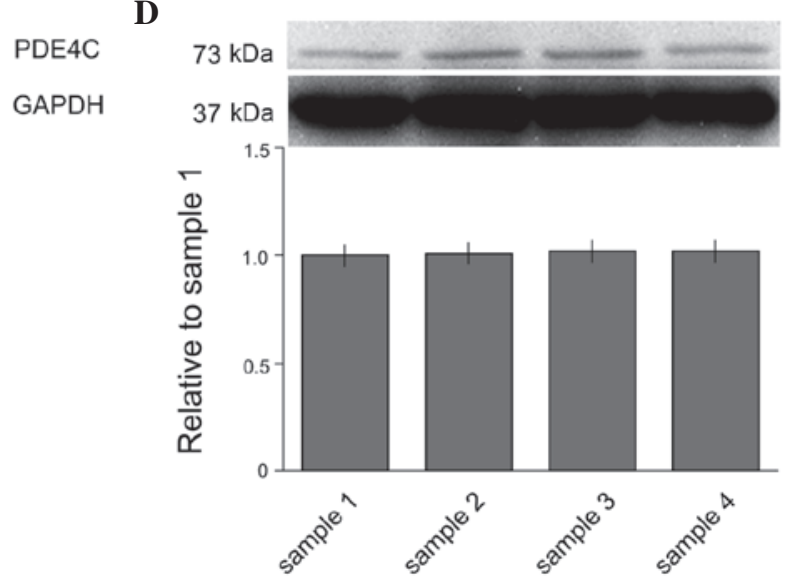

PDE4B

GAPDH

PDE4D

GAPDH

Figure 1. Expression of (A) PDE4A, (B) PDE4B, (C) PDE4C and (D) PDE4D in microglia. Microglia expressed all four subtypes of PDE4. PDE, phosphodiesterase; GAPDH, glyceraldehyde 3-phosphate dehydrogenase.

\section{Discussion}

In this study, the expression of PDE4 subtypes A-D was detected in primary microglia at the protein level. Transfection of siRNA silenced the expression of all four subtypes of PDE4 in microglial cells. However, the significantly elevated levels of proinflammatory cytokines TNF- $\alpha$ and IL- $1 \beta$ could only be suppressed by silencing $\mathrm{PDE} 4 \mathrm{~B}$, and not by silencing PDE4A, PDE4C or PDE4D. The significantly decreased level of cAMP induced by LPS stimulation could only be elevated by silencing PDE4B, and not by silencing PDE4A, PDE4C or PDE4D. Furthermore, the shift from ERK to p-ERK that was induced by LPS stimulation was inhibited by the silencing of PDE4B.

These results suggest that, among the four subtypes of PDE4 only PDE4B plays a pivotal role in regulating the inflammatory response in microglia. This result was consistent with previous studies. Jin et al suggested that ablation of PDE4B, but not PDE4A or PDE4D, significantly suppressed LPS-induced TNF- $\alpha$ production in circulating monocytes and peritoneal macrophages $(24,25)$. Robichaud et al advised that inhibition of PDE4D but not PDE4B may be responsible for the emetic effects of non-selective PDE4 inhibitors; the reason may be that in the CNS, PDE4D is linked to cAMP signaling of the 2-adrenoceptor in noradrenergic neurons (26).
In comparison with previous studies, however, attention was paid to several important points. To explore the detailed functions of the four subtypes, RNAi was selected to knock down the expression of PDE4A, PDE4B, PDE4C and PDE4D, respectively. RNAi is a straightforward method to eliminate the function of any gene of interest.

Furthermore, in this study, the intracellular cAMP level was measured by ELISA. The results demonstrated that compared with the LPS group, PDE4B silencing markedly enhanced cAMP accumulation, while silencing of the other PDE4 subtypes did not significantly affect the cAMP level in microglial cells, suggesting that PDE4B plays a pivotal role in the regulation of the cAMP level in microglial cells.

cAMP is a key intracellular second messenger, and PDE4B siRNA would prevent PDE4B from hydrolyzing cAMP, thus maintaining intracellular cAMP levels and providing an increased stabilizing effect since they are significantly reduced following LPS stimulation. Activation of the membrane G-protein coupled receptor subunit Gsa causes cAMP levels to increase, which inhibits the inflammatory response (27). Gsa activates adenylyl cyclase, an enzyme with numerous downstream effector systems associated with cell signaling. Rac activity is downregulated via the cAMP-protein kinase A pathway, which leads to reduced neutrophil migration (28-30). Increased cAMP levels reduce the expression of adhesion molecules (30) and 


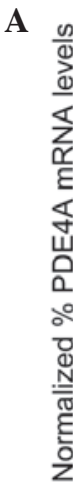

C

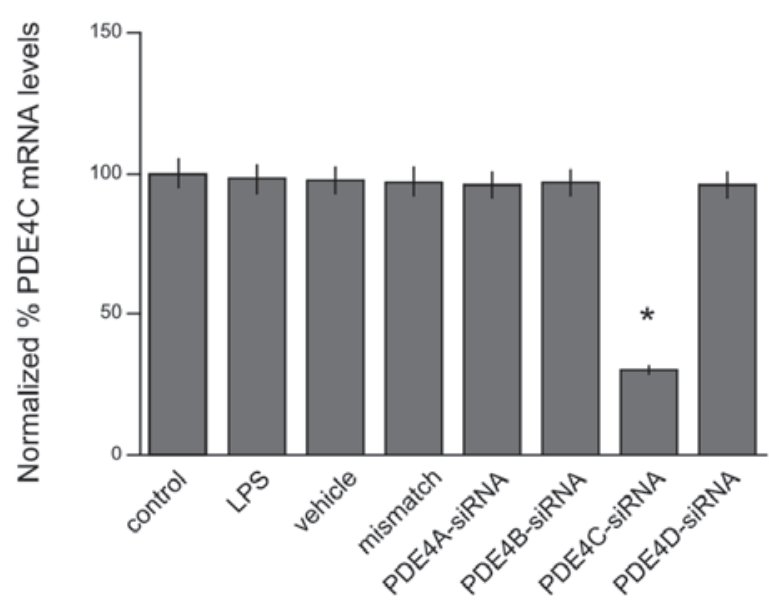

B

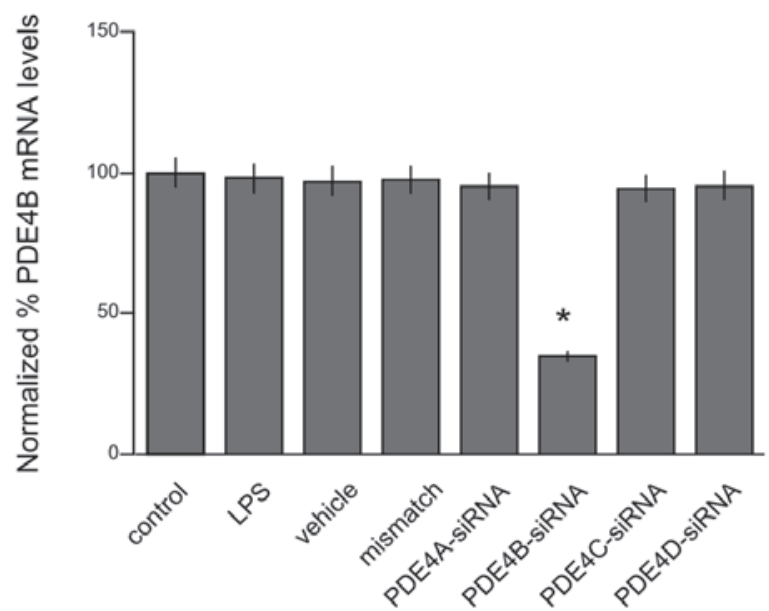

D

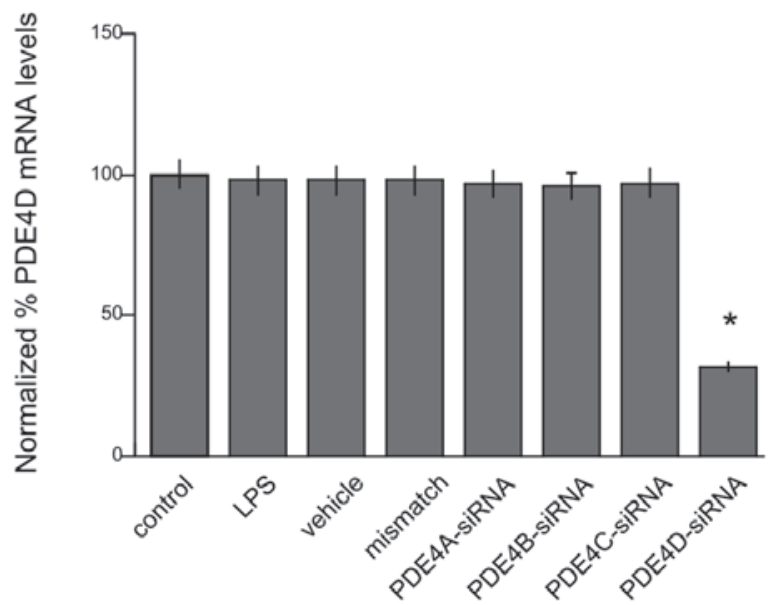

Figure 2. Reduced expression levels of PDE4A, PDE4B, PDE4C and PDE4D mRNA by siRNAs. (A) The expression of PDE4A at the mRNA level was reduced to $36.6 \pm 5.2 \%$ of that in the control group ("P<0.05). The expression of (B) PDE4B, (C) PDE4C and (D) PDE4D at the mRNA level was reduced to 34.7 \pm 6.4 , $33.2 \pm 4.8$ and $27.5 \pm 7.0 \%$ of that in the control group, respectively ( $\left.{ }^{*} \mathrm{P}<0.05\right)$. However, no significant differences were observed among the LPS, mismatch RNA and vehicle groups $(\mathrm{P}>0.05)$. PDE, phosphodiesterase; siRNA, small interfering RNA; LPS, lipopolysaccharide.
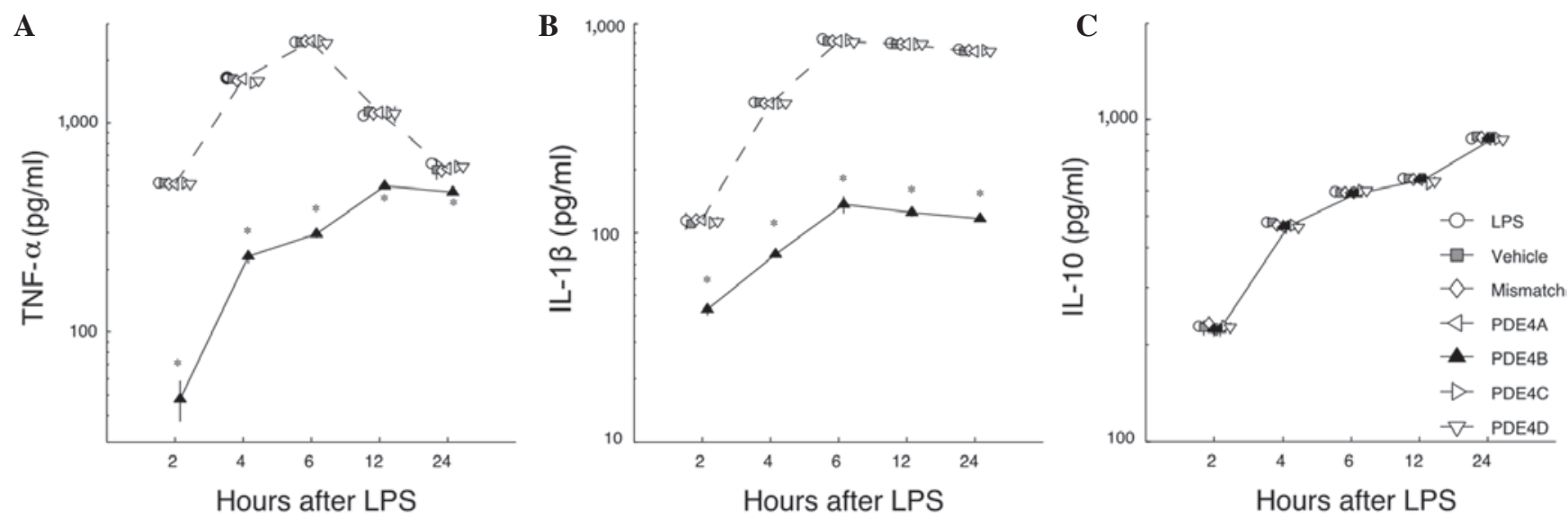

Figure 3. Influence of siRNAs of PDE4A, PDE4B, PDE4C and PDE4D on the LPS-induced release of TNF- $\alpha$, IL-1 $\beta$ and IL-10 in microglia. (A) Expression of TNF- $\alpha$. Among the groups treated with siRNAs only the PDE4B group showed significantly reduced levels of TNF- $\alpha$ compared with the LPS group ("P<0.05). (B) Expression of IL-1 $\beta$. Among the groups treated with siRNAs, only PDE4B group showed significantly reduced levels of IL-1 $\beta$ compared with the LPS group ("P $<0.05)$. (C) Expression of IL-10. Levels of IL-10 were not affected by any of siRNAs compared with LPS group (P $>0.05)$. PDE, phosphodiesterase; siRNA, small interfering RNA; LPS, lipopolysaccharide; TNF, tumor necrosis factor; IL, interleukin.

disrupt chemokine-induced chemotaxis (31). High cAMP levels also inhibit leukocyte adhesion and migration (32), and downregulate phagocytosis and nitric oxide production in macrophages (33). 


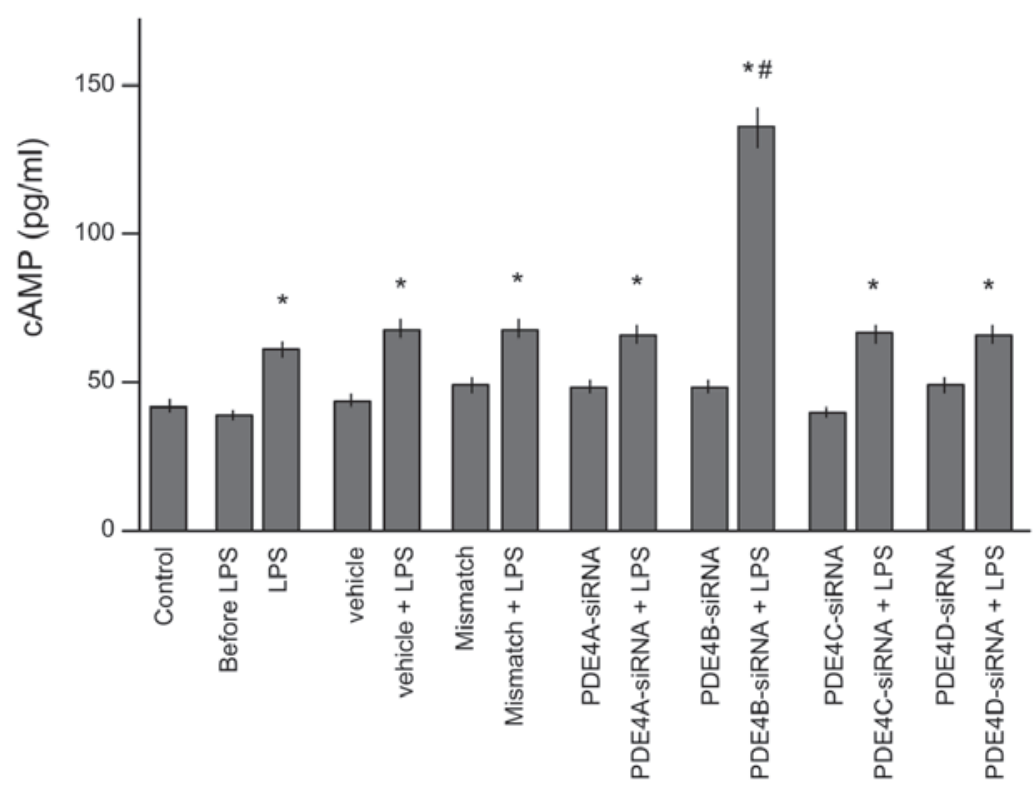

Figure 4. Effects of PDE4A, PDE4B, PDE4C and PDE4D siRNA on the intracellular cAMP level in microglia. LPS stimulation significantly increased the cAMP level in all groups compared with the control group (" $\mathrm{P}<0.05)$. However, only the PDE4B-siRNA group exhibited a significantly higher intracellular cAMP level than the LPS group $\left({ }^{*} \mathrm{P}<0.05\right)$. PDE, phosphodiesterase; siRNA, small interfering RNA; LPS, lipopolysaccharide; cAMP, cyclic adenosine monophosphate.

A
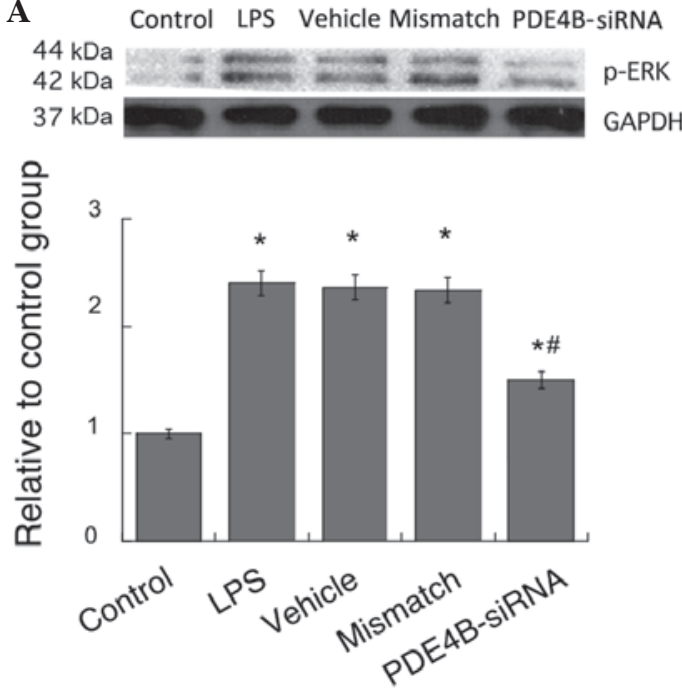

B Control LPS Vehicle Mismatch PDE4B-siRNA
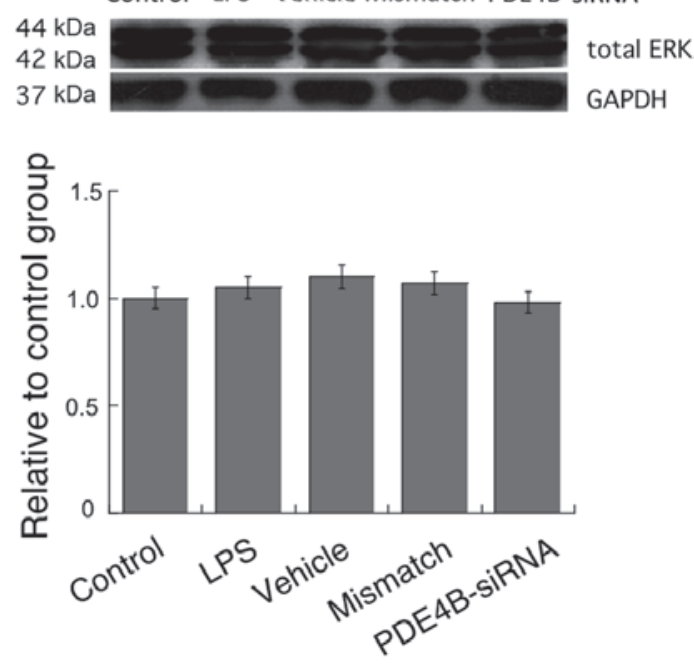

Figure 5. Effect of PDE4B-siRNA on the activation of ERK induced by LPS in microglia. (A) Expression of p-ERK. Compared with the control group, $\mathrm{p}$-ERK levels in the other four groups were significantly increased after LPS stimulation ( $\mathrm{P}<0.05)$. Only the PDE4B-siRNA group showed a decreased p-ERK level compared with the LPS group $\left({ }^{*} \mathrm{P}<0.05\right)$. (B) Expression of total ERK. There were no significant differences in total ERK levels among all groups $(\mathrm{P}>0.05)$. PDE, phosphodiesterase; siRNA, small interfering RNA; LPS, lipopolysaccharide; ERK, extracellular signal-regulated kinase.

It has been suggested that cAMP signaling is regulated by an ERK-dependent pathway. ERK can influence the activities of numerous transcriptional factors including cAMP response element-binding protein and nuclear factor- $\kappa \mathrm{B}(34,35)$. Zhuang et al suggested that there is robust activation of ERK in microglia (36). The intensity of ERK activation is closely correlated with activation of glial cells. It phosphorylates many downstream targets that lead to inhibition of cell activation (36). In the present study, p-ERK intensity was greatly increased by LPS stimulation compared with the control group. The p-ERK protein expression in the PDE4B-silenced group was decreased significantly compared with that in the LPS group. The total ERK level did not differ significantly among the groups.

The present data about TNF- $\alpha$, IL-1 $\beta$ and IL-10 are consistent with previous findings and support the increased cAMP concentration. Considering these findings together, the activation of the ERK/MARK pathway may lead to the release of large quantities of pro-inflammatory cytokines from the microglial cells.

The present study provides solid evidence that PDE4B in microglial cells plays an important role in neuropathic pain associated with inflammatory responses. Clinically, a selective 
PDE inhibitor targeting PDE4B could be a promising candidate in a new therapeutic strategy for neuropathic pain.

\section{References}

1. Gremo F, Sogos V, Ennas MG, Meloni A, Persichini T, Colasanti $\mathrm{M}$ and Lauro GM: Features and functions of human microglia cells. Adv Exp Med Biol 429: 79-97, 1997.

2. Wyss-Coray $\mathrm{T}$ and Rogers J: Inflammation in Alzheimer disease - a brief review of the basic science and clinical literature. Cold Spring Harb Perspect Med 2: a006346, 2012.

3. Karagkouni A, Alevizos M and Theoharides TC: Effect of stress on brain inflammation and multiple sclerosis. Autoimmun Rev 12: 947-953, 2013.

4. Campbell JN and Meyer RA: Mechanisms of neuropathic pain. Neuron 52: 77-92, 2006.

5. Aloisi F: Immune function of microglia. Glia 36: 165-179, 2001.

6. Garden GA and Möller T: Microglia biology in health and disease. J Neuroimmune Pharmacol 1: 127-137, 2006.

7. Buttini M, Mir A, Appel K, Wiederhold KH, Limonta S, Gebicke Haerter PJ and Boddeke HW: Lipopolysaccharide induces expression of tumour necrosis factor alpha in rat brain: Inhibition by methylprednisolone and by rolipram. Br J Pharmacol 122: 1483-1489, 1997.

8. Tanaka J, Toku K, Matsuda S, Sudo S, Fujita H, Sakanaka M and Maeda N: Induction of resting microglia in culture medium devoid of glycine and serine. Glia 24: 198-215, 1998.

9. Zhang B, Yang L, Konishi Y, Maeda N, Sakanaka M and Tanaka J: Suppressive effects of phosphodiesterase type IV inhibitors on rat cultured microglial cells: comparison with other types of cAMP-elevating agents. Neuropharmacology 42: 262-269, 2002

10. Conti M and Jin SL:The molecular biology of cyclic nucleotide phosphodiesterases. Prog Nucleic Acid Res Mol Biol 63: 1-38, 1999.

11. Houslay MD: Underpinning compartmentalised cAMP signalling through targeted cAMP breakdown. Trends Biochem Sci 35: 91-100, 2010.

12. Jarnagin K, Chanda S, Coronado D, Ciaravino V, Zane LT, Guttman-Yassky E and Lebwohl MG: Crisaborole topical ointment, 2\%: A nonsteroidal, topical, anti-inflammatory phosphodiesterase 4 inhibitor in clinical development for the treatment of atopic dermatitis. J Drugs Dermatol 15: 390-396, 2016.

13. Pearse DD and Hughes ZA: PDE4B as a microglia target to reduce neuroinflammation. Glia: Apr 1, 2016 doi: 10.1002/glia.22986 (Epub ahead of print).

14. Flemming S, Schlegel N, Wunder C, Meir M, Baar W, Wollborn J, Roewer N, Germer CT and Schick MA: Phosphodiesterase 4 inhibition dose dependently stabilizes microvascular barrier functions and microcirculation in a rodent model of polymicrobial sepsis. Shock 41: 537-545, 2014.

15. Jin SL, Ding SL and Lin SC: Phosphodiesterase 4 and its inhibitors in inflammatory diseases. Chang Gung Med J 35: 197-210, 2012

16. Fire A, Xu S, Montgomery MK, Kostas SA, Driver SE and Mello CC: Potent and specific genetic interference by double-stranded RNA in Caenorhabditis elegans. Nature 391: 806-811, 1998

17. Doré-Savard L, Roussy G, Dansereau MA, Collingwood MA, Lennox KA, Rose SD, Beaudet N, Behlke MA and Sarret P: Central delivery of Dicer-substrate siRNA: A direct application for pain research. Mol Ther 16: 1331-1339, 2008.

18. Röhl T and Kurreck J: RNA interference in pain research. J Neurochem 99: 371-380, 2006.

19. Jiang H, Bielekova B, Okazaki H, Clarence-Smith K, Johnson KP, Bergey G, Martin R and Dhib-Jalbut S: The effect of vesnarinone on TNF alpha production in human peripheral blood mononuclear cells and microglia: A preclinical study for the treatment of multiple sclerosis. J Neuroimmunol 97: 134-145, 1999.
20. Xue Y, Wang Y, Feng DC, Xiao BG and Xu LY: Tetrandrine suppresses lipopolysaccharide-induced microglial activation by inhibiting NF-kappaB pathway. Acta Pharmacol Sin 29: 245-251, 2008.

21. Liu J, Zhao X, Cao J, Xue Q, Feng X, Liu X, Zhang F and Yu B: Differential roles of PKA and Epac on the production of cytokines in the endotoxin-stimulated primary cultured microglia. J Mol Neurosci 45: 186-193, 2011.

22. Choi DC, Lee JY, Lim EJ, Baik HH, Oh TH and Yune TY: Inhibition of ROS-induced p38MAPK and ERK activation in microglia by acupuncture relieves neuropathic pain after spinal cord injury in rats. Exp Neurol 236: 268-282, 2012.

23. Li W, Li Y, Zhu S, Ji Q, Shu Y, Zhang L and Liu J: Rosuvastatin attenuated the existing morphine tolerance in rats with L5 spinal nerve transection through inhibiting activation of astrocytes and phosphorylation of ERK42/44. Neurosci Lett 584: 314-319, 2015.

24. Jin SL and Conti M: Induction of the cyclic nucleotide phosphodiesterase PDE4B is essential for LPS-activated TNF-alpha responses. Proc Natl Acad Sci USA 99: 7628-7633, 2002

25. Jin SL, Lan L, Zoudilova M and Conti M: Specific role of phosphodiesterase 4B in lipopolysaccharide-induced signaling in mouse macrophages. J Immunol 175: 1523-1531, 2005.

26. Robichaud A, Stamatiou PB, Jin SC, Lachance N, MacDonald D, Laliberté F, Liu S, Huang Z, Conti M and Chan CC: Deletion of phosphodiesterase 4D in mice shortens alpha(2)-adrenoceptor-mediated anesthesia, a behavioral correlate of emesis. J Clin Invest 110: 1045-1052, 2002.

27. Bao F, Fleming JC, Golshani R, Pearse DD, Kasabov L, Brown A and Weaver LC: A selective phosphodiesterase-4 inhibitor reduces leukocyte infiltration, oxidative processes and tissue damage after spinal cord injury. J Neurotrauma 28: 1035-1049, 2011.

28. Erdogan S, Aslantas O, Celik S and Atik E: The effects of increased cAMP content on inflammation, oxidative stress and PDE4 transcripts during Brucella melitensis infection. Res Vet Sci 84: 18-25, 2008.

29. Pearse DD, Pereira FC, Marcillo AE, Bates ML, Berrocal YA, Filbin MT and Bunge MB: cAMP and Schwann cells promote axonal growth and functional recovery after spinal cord injury. Nat Med 10: 610-616, 2004.

30. Nagasawa SY, Takuwa N, Sugimoto N, Mabuchi H and Takuwa Y: Inhibition of Rac activation as a mechanism for negative regulation of actin cytoskeletal reorganization and cell motility by cAMP. Biochem J 385: 737-744, 2005.

31. Sanz MJ, Cortijo J and Morcillo EJ: PDE4 inhibitors as new anti-inflammatory drugs: Effects on cell trafficking and cell adhesion molecules expression. Pharmacol Ther 106: 269-297, 2005.

32. Lorenowicz MJ, Fernandez-Borja M and Hordijk PL: cAMP signaling in leukocyte transendothelial migration. Arterioscler Thromb Vasc Biol 27: 1014-1022, 2007.

33. Ninković J and Roy S: Morphine decreases bacterial phagocytosis by inhibiting actin polymerization through cAMP-, Rac-1- and p38 MAPK-dependent mechanisms. Am J Pathol 180: 1068-1079, 2012.

34. Emery AC and Eiden LE: Signaling through the neuropeptide GPCR PAC ${ }_{1}$ induces neuritogenesis via a single linear cAMPand ERK-dependent pathway using a novel cAMP sensor. FASEB J 26: 3199-3211, 2012.

35. Enserink JM, Christensen AE, de Rooij J, van Triest M, Schwede F, Genieser HG, Døskeland SO, Blank JL and Bos JL: A novel Epac-specific cAMP analogue demonstrates independent regulation of Rap1 and ERK. Nat Cell Biol 4: 901-906, 2002.

36. Zhuang ZY, Gerner P, Woolf CJ and Ji RR: ERK is sequentially activated in neurons, microglia and astrocytes by spinal nerve ligation and contributes to mechanical allodynia in this neuropathic pain model. Pain 114: 149-159, 2005. 